\title{
Efficient Solution Technique for Solving the Poisson-Boltzmann Equation
}

\author{
ABDALLAH SAYYED-AHMAD, KAGAN TUNCAY, PETER J. ORTOLEVA \\ Center for Cell and Virus Theory, Department of Chemistry, Indiana University, \\ Bloomington, Indiana 47405
}

Received 9 October 2003; Accepted 9 February 2004

\begin{abstract}
The Poisson-Boltzmann (PB) equation has been extensively used to analyze the energetics and structure of proteins and other significant biomolecules immersed in electrolyte media. A new highly efficient approach for solving PB-type equations that allows for the modeling of many-atoms structures such as encountered in cell biology, virology, and nanotechnology is presented. We accomplish these efficiencies by reformulating the elliptic PB equation as the long-time solution of an advection-diffusion equation. An efficient modified, memory optimized, alternating direction implicit scheme is used to integrate the reformulated PB equation. Our approach is demonstrated on protein composites (a polio virus capsid protomer and a pentamer). The approach has great potential for the analysis of supramillion atoms immersed in a host electrolyte.
\end{abstract}

(C) 2004 Wiley Periodicals, Inc. J Comput Chem 25: 1068-1074, 2004

Key words: Poisson-Boltzmann; electrostatics; finite difference method

\section{Introduction}

Electrostatic interactions play a crucial role in determining the structure and behavior of proteins and more complex structures (e.g., enzymes and viruses). For example, DNA is overall negatively charged due to phosphate groups. Biomolecules reside in an aqueous electrolyte, which affects their conformation and function due to screening and dielectric effects. Aqueous physiological media contain many mobile ions (e.g., $\mathrm{Cl}^{-}, \mathrm{Na}^{+}, \mathrm{K}^{+}, \mathrm{Mg}^{++}$, and $\mathrm{Ca}^{++}$), which redistribute to screen the Coulomb potential of the fixed charges on the macromolecules by creating layers of counterions. Furthermore, orientable or polarizable molecules of the host medium also serve to decrease the electrical forces that determine structure and function of macromolecules. Accurate calculation of the electrostatic potential can enhance our understanding of the behavior and structure of macromolecules. ${ }^{1}$ One can use such calculations to estimate the solvation energy, find $\mathrm{pKa}$ values, ${ }^{2}$ and titration curves for proteins. Also, one can calculate the electrostatic forces between biomolecules for use in molecular dynamics. ${ }^{3}$ In carrying out molecular mechanics computations, it is important to account for the channeling of the electric field along a macromolecule due to the dielectric constant contrast between the aqueous medium and the interior of a protein, an effect not accounted for in $1 / r$ Coulomb computations as in the CHARMM force field.

The rigorous statistical mechanical theory of Coulomb systems is fraught with challenges due to the long range of the interparticle potential. The starting point is the determination of the quantum energy states of the electron-nucleus system. From the BornOppenheimer approximation, one arrives at an effective N-body potential for the nuclei when the electronic system can be assumed to be in the ground state. Thus, the problem is reduced to the statistical mechanics of many nuclei evolving in the Born-Oppenheimer potential. As a practical approach, phenomenological formulations based on heuristic arguments based on classical continuum electrostatics and equilibrium statistical mechanics are usually used to compute the electrostatic potential around a molecule immersed in an electrolyte. In such an approach, the host medium consists of water molecules and mobile ions accounted for by using macroscopic continuum densities.

The PB equation has been traditionally used to find the electrostatic potential around a macromolecule. A PB model ignores the volume of ions in the medium. Therefore, $\mathrm{PB}$ equation is valid for dilute ionic solutions with several Debye lengths away from the fixed charges on the mesostructure of interest (i.e., concentration $\leq 0.15 \mathrm{M}){ }^{4}$ This model has been applied to calculate properties of molecules in solution. ${ }^{1}$ Extensions of the PB model to

\footnotetext{
Correspondence to: P. J. Ortoleva; e-mail: Ortoleva@Indiana.edu Contract/grant sponsors: U.S. Air Force (Defense Advanced Research Projects Agency), the U.S. Department of Energy, the Indiana 21st Century Science and Technology Fund, IBM (Shared University Research grants to Indiana University), and IBM Life Sciences Institutes of Innovations Program.
} 
account for the mobile ions sizes and correlations have been developed. ${ }^{5,6}$ The PB model accounts for solvent molecules implicitly via the dielectric constant $\varepsilon ; \varepsilon$ is low within the molecule, and is assumed to increase gradually to the unperturbed, far-field value over several angstroms away from the molecule of interest. ${ }^{7}$ Solutions to simple problems with spherical, cylindrical, or planar symmetry are available for the linearized PB equation. ${ }^{8,9}$ A closedform formula for the solution of nonlinear PB does not seem to be possible except for the planar case ${ }^{10}$ and the infinite cylindrical symmetric case, where only counterions exist in the solution. ${ }^{11}$

In attempting to use numerical methods to solve the PB equation with complicated molecular geometries and charge distributions, a number of challenges present themselves. The equation is highly nonlinear due to the exponential dependence of the mobile ion concentrations on the potential. The electrical potential varies on more than one length scale. The associated characteristic lengths are the size of an atom, the Debye length, and the size of the structure of interest. In addition, the length required for the dielectric constant to reach its far field value has a considerable effect on the potential (see Spatial Variations section). In summary, one must solve highly nonlinear, multiple scale equations with thousands to millions of spatial variations when addressing macromolecules and other mesoscopic systems.

Several approaches and numerical codes to solve the linear and nonlinear PB equation have been presented throughout the last decade. An overview of numerical techniques commonly applied to the PB type equations is provided in ref. 12. For the linear PB, a basis set approach is used to express the electrostatic potential as a linear superposition of basis functions. ${ }^{13}$ The electrostatic energy functional is then minimized with respect to the expansion coefficients subject to total charge conservation. The boundary element method utilizes analytical solutions obtained in terms of Green's functions and discretization on the domain surface (molecular surface) are used to compute the potential in the whole domain volume. ${ }^{14,15}$ One of the most common approaches used to solve the linear and the nonlinear PB is the finite difference formulation where spatial derivatives are approximated using neighboring points. ${ }^{16-18}$ A successive overrelaxation method has been used to get rapid convergence in solving the linear systems obtained from the finite difference discretization. ${ }^{16}$ Recently, the finite element method has been successfully used to solve the PB equation for larger systems. An adaptive multilevel approach based on tetrahedral elements to create a dense mesh to capture the dielectric discontinuity across the molecular surface has been also used. Although irregular grids can be used in a finite element approach, it is inherently less accurate than regular grid methods due to neighboring large and small elements (unless a gradual increase in the element size is imposed). ${ }^{19}$ Furthermore, one might argue that in a physically relevant model, the dielectric discontinuity is not present; rather, $\varepsilon$ gradually increases to its unperturbed bulk value with distance from the molecule-medium boundary. ${ }^{18}$ Also, regular grids lead to simpler and higher order numerical schemes and they allow implementation of multigrid methods. ${ }^{20}$ Tomac and Gräslund $^{21}$ demonstrated a multigrid finite volume method for solving a modified PB equation for spherical charged particles. The modified PB equation agrees with the results obtained from Monte Carlo simulations on 1:1 and 2:1 ionic solutions. A pseudotransient continuation and finite element $\operatorname{method}^{22}$ has been adapted to solve the nonlinear PB equation to utilize the radiationhydrodynamic parallel code ICF3D. The approach was demonstrated to give accurate solutions for spherically symmetric systems.

This study is motivated by the need to have fine meshes to resolve the structure of, and the potential distribution around, large macromolecules and their complexes. A novel numerical approach is developed to reduce the memory requirement by approximately one order of magnitude in comparison to a Galerkin finite element method. The solution of the nonlinear PB equation is obtained from the time evolution of a diffusion-advection equation where there is no need to use Newton-Raphson-like methods. A verification of the solution obtained from our approach is accomplished by comparison with the solution of a charged spherical particle. Results on the polio virus protomer and pentamer illustrate the viability of our approach for large systems.

\section{Formulation}

To begin, we briefly review the derivation of the PB model. ${ }^{23,24}$ Poisson's equation for the electrostatic potential $\varphi$, which is related to the work needed to assemble the charge density $\rho$, is given by

$$
\vec{\nabla} \cdot(\varepsilon \vec{\nabla} \varphi)=-4 \pi \rho .
$$

The relative dielectric constant $\varepsilon$ is equal to $1\left(\varepsilon_{\text {vacuum }}=\right.$ $8.854187817 \times 10^{-12} \mathrm{Coul} / \mathrm{Nm}^{2}$ in SI units) for vacuum, and approximately 80 for liquid water at room temperature. The boundary conditions are

$$
\lim _{r \rightarrow \infty} \varphi(r)=0
$$

However, for a finite computational domain, $\varphi$ is commonly truncated at a distance where the potential is expected to be negligible.

The dynamics of mobile ions is highly correlated. If an ion moves, the surrounding mobile ions will move to attain charge neutrality. A rigorous approach should include the thermal motion of strongly interacting many-body systems of ions, but such an approach is computationally difficult. The charge density surrounding a macromolecule is neither uniform nor static. Because the folding of proteins and many other biological processes occur on a time scale longer than the relaxation time to reorganize a mobile counterion charge cloud. In fact, the mobile ions charge density is usually assumed to adiabatically follow the conformation of the macromolecule or other structure of interest.

Consider a macromolecule (a protein) immersed in an electrolyte. Assume that a the structure of the protein is known with some fixed charges $q_{i}$ obtained from a parameterized force field (e.g., CHARMM22 ${ }^{25}$ ). The electrolyte is composed of ionic species with bulk concentrations $c_{i}^{\infty}$ (i.e., far from the embedded molecule). With this the total free charge density is given by

$$
\rho(\vec{r}, \underline{c})=\rho_{\text {ions }}+\rho_{\text {macromolecule }}=F \sum_{i=1}^{N_{\text {ions }}} z_{i} c_{i}(\vec{r})+\sum_{i=1}^{N_{\text {charges }}} q_{i} \delta\left(\vec{r}-\vec{r}_{i}\right),
$$



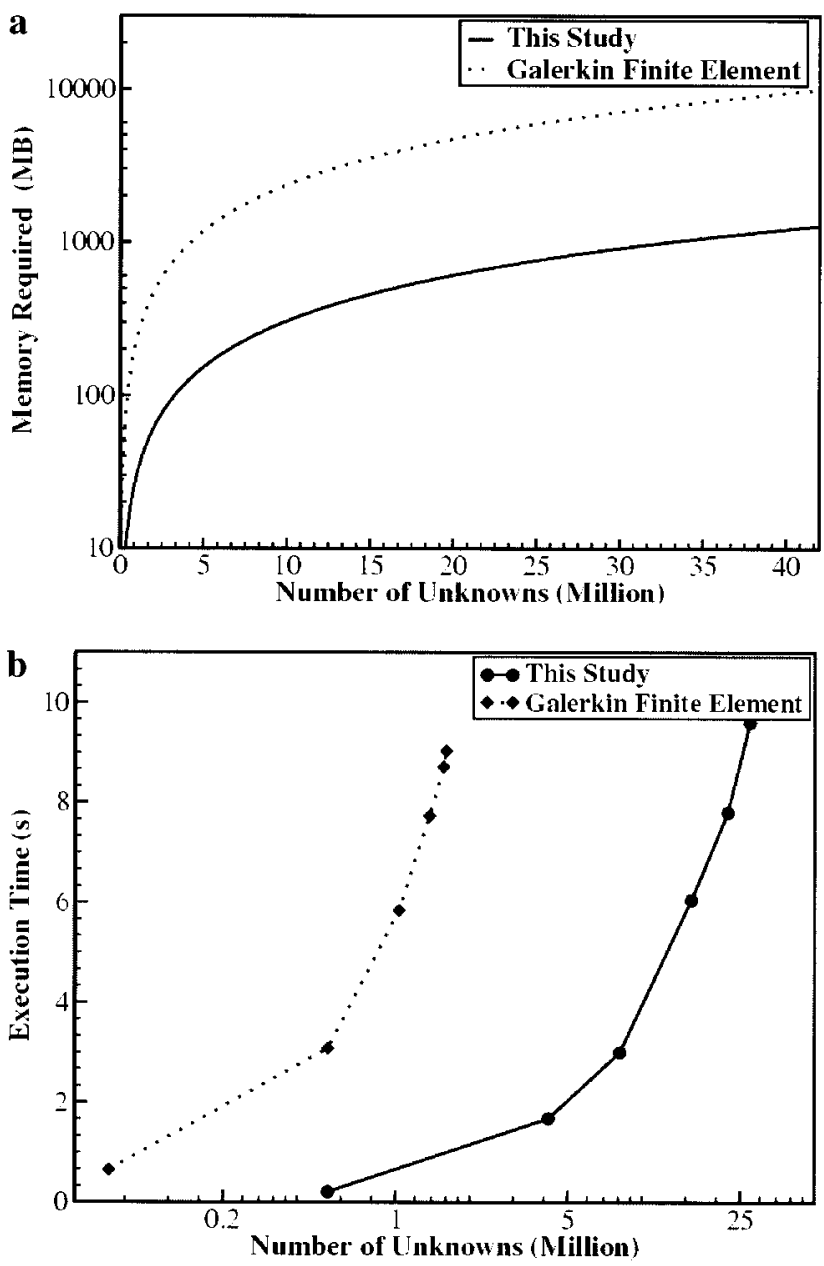

Figure 1. (a) Comparison of memory requirements between the Galerkin finite element approach and our method. (b) Comparison of measured execution time between the Galerkin finite element approach (Newton-Raphson step) and our method (steepest descent time step).

where $F$ is Faraday's constant, $z_{i}$ and $c_{i}$ are the valence and concentration of mobile ion type $i$, and $\vec{r}_{i}$ is the position of the $i$ th fixed charge on the structure.

To obtain the concentrations for each ionic species, the DebyeHuckel theory uses the equilibrium thermodynamic relation for the chemical potential of a dilute electrolyte ${ }^{26}$

$$
\mu_{i}=\mu_{i}^{*}+R T \ln c_{i}(\vec{r})+z_{i} F \varphi(\vec{r}) .
$$

The above equation establishes the relationship between the electrostatic potential and the concentrations of the mobile ions. In the above, high concentrations and finite ions size effects are ignored. The simplest extension needed to account for these would be to use a van der Waals-like equation of state or empirically calibrated chemical potentials. For the demonstration of our numerical approach, a dilute solution theory is adopted. However, in the dilute limit (4) yields

$$
c_{i}(\vec{r})=c_{i}^{\infty} \exp \left(-z_{i} F \varphi(\vec{r}) / R T\right)
$$

where $c_{i}^{\infty}$ is the bulk concentration of ionic species $i$. The above equation establishes a direct nonlinear relationship (Boltzmann distribution) between the electrostatic potential and the mobile ion concentrations. Substitution of (5) and (2) in (1) results in the nonlinear Poisson-Boltzmann equation

$$
\begin{aligned}
\vec{\nabla} \cdot(\varepsilon(\vec{r}) \vec{\nabla} \varphi)+4 \pi F \sum_{i=1}^{N_{\text {ions }}} z_{i} c_{i}^{\infty} \exp ( & \left.-z_{i} F \varphi(\vec{r}) / R T\right) \\
& +4 \pi \sum_{i=1}^{N_{\text {charges }}} q_{i} \delta\left(\vec{r}-\vec{r}_{i}\right)=0 .
\end{aligned}
$$

This equation serves as the basis for the demonstration of our numerical approach.

\section{Numerical Approach}

Solving the elliptic PB equation in 3D is computationally intensive. To capture the molecule-medium interface, and distinguish the structure of the macromolecule from the surrounding, one needs sufficiently fine grids (at least 0.5 Angstrom). Solution of (6) using the finite element method on a hexahedral grid with conjugate gradient iterative solver with compressed coefficient matrix approximately requires $250 \mathrm{~N}$ bytes; where $N$ is the number of nodes in the mesh. This translates to a limiting resolution of $\sim 160^{3}$ on a 1 GB RAM computer (Fig. 1a, Table 1).

We formulate a nonlinear advection-diffusion equation using the original elliptic nonlinear PB equation as follows. We start with the variational functional formulation for the elliptic PB equation

$\Xi[\varphi]=\int_{\Omega} d^{3} r\left(\frac{1}{2} \varepsilon(r)|\nabla \varphi(r)|^{2}-4 \pi \int_{0}^{\varphi(r)} d \varphi^{\prime} \rho\left(r, \varphi^{\prime}\right)\right)$.

The PB equation is obtained by minimizing (7) with respect to $\varphi$; provided that $\varphi$ or its derivatives vanishes on the boundaries of the domain $\Omega$. The functional $\Xi$ is minimized when its first variation vanishes, i.e.,

Table 1. Memory Requirement for Solving PB Equation on a Regular (PMG) and Adaptive Grid (MC) Finite Element. ${ }^{29}$

\begin{tabular}{lrrr}
\hline Solver & \# Vertices & \# Cells & Memory \\
\hline PMG & $2,146,689$ & $2,097,152$ & $515 \mathrm{MB}$ \\
MC & 67,288 & 373,978 & $109 \mathrm{MB}$ \\
This study & $2,146,689$ & $2,097,152$ & $65 \mathrm{MB}$ \\
This study & $27,270,901$ & $27,000,000$ & $832 \mathrm{MB}$
\end{tabular}

Our approach requires one order of magnitude less memory than PMG for the same number of unknowns. 


$$
\frac{\delta \Xi}{\delta \varphi}=0 .
$$

We use a Langevin steepest descent approach with friction coefficient $\varepsilon^{-1}$ to minimize the functional $\Xi$

$$
\varepsilon(\vec{r}) \frac{\partial \varphi}{\partial t}=-\frac{\delta \Xi}{\delta \varphi} .
$$

Calculating the functional derivative $\Xi$ with respect to $\varphi$ and substituting in (9) yields

$$
\varepsilon \frac{\partial \varphi}{\partial t}=\nabla \cdot(\varepsilon \nabla \varphi)+4 \pi \rho,
$$

which can be manipulated to obtain

$$
\begin{aligned}
\frac{\partial \varphi}{\partial t}=\nabla^{2} \varphi+\frac{\nabla \varepsilon \cdot \nabla \varphi}{\varepsilon}+\frac{4 \pi F}{\varepsilon} \sum_{i=1}^{N_{\text {ions }}} z_{i} c_{i}^{\infty} & \exp \left(-z_{i} F \varphi / R T\right) \\
& +\frac{4 \pi}{\varepsilon} \sum_{i=1}^{N_{\text {charges }}} q_{i} \delta\left(\vec{r}-\vec{r}_{i}\right)
\end{aligned}
$$

Because the PB equation has a unique solution, the steady-state solution of the above advection-diffusion equation corresponds to the solution of the elliptic PB equation. Despite the simplicity and the modest storage requirements of an explicit forward in time, centered in space (FTCS), the integration scheme for solving the advection-diffusion equation, using the FTCS scheme, is not efficient as the stability of the time integration is constrained by Courant and Peclet numbers. ${ }^{19}$ Therefore, one needs a large number of time steps to reach the steady-state solution. On the other hand, although a fully implicit scheme allows one to use much larger time steps, it is memory demanding because of the need to store a large sparse matrix. To get the advantages of both techniques we adapt an operator splitting scheme. A commonly used splitting algorithm is alternating direction method (ADI), which is a variation of Crank-Nicholson scheme. ${ }^{27}$

The 3D Douglas ADI for the reformulated PB equation has three steps

$$
\begin{aligned}
&\left(1-\frac{1}{2} r_{x} \delta_{x}^{2}\right)\left(\varphi_{i j k}^{n+1 / 3}-\right.\left.\varphi_{i j k}^{n}\right)=\left(r_{x} \delta_{x}^{2}+r_{y} \delta_{y}^{2}+r_{z} \delta_{z}^{2}\right) \varphi_{i j k}^{n}+\Delta t \tilde{\rho}_{i j k}^{n} \\
&\left(\varphi_{i j k}^{n+2 / 3}-\varphi_{i j k}^{n+1 / 3}\right)=\frac{1}{2} r_{y} \delta_{y}^{2}\left(\varphi_{i j k}^{n+2 / 3}-\varphi_{i j k}^{n}\right) \\
& \times\left(\varphi_{i j k}^{n+1}-\varphi_{i j k}^{n+2 / 3}\right)=\frac{1}{2} r_{z} \delta_{z}^{2}\left(\varphi_{i j k}^{n+1}-\varphi_{i j k}^{n}\right),
\end{aligned}
$$

where

$\tilde{\rho}_{i j k}^{n}=\frac{1}{\varepsilon_{i j k}}\left(\delta_{x} \varepsilon_{i j k} \delta_{x} \varphi_{i j k}^{n}+\delta_{y} \varepsilon_{i j k} \delta_{y} \varphi_{i j k}^{n}+\delta_{z} \varepsilon_{i j k} \delta_{z} \varphi_{i j k}^{n}\right)+\frac{4 \pi}{\varepsilon_{i j k}} \rho_{i j k}^{n}$,

and

$$
r_{x}=\frac{\Delta t}{\Delta x^{2}} \quad r_{y}=\frac{\Delta t}{\Delta y^{2}} \quad r_{z}=\frac{\Delta t}{\Delta z^{2}}
$$

The centered finite difference operators are defined as

$$
\begin{aligned}
& \Delta x^{2} \delta_{x}^{2} \varphi_{i j k}=\varphi_{i+1 j k}-2 \varphi_{i j k}+\varphi_{i-1 j k} \\
& \Delta y^{2} \delta_{y}^{2} \varphi_{i j k}=\varphi_{i j+1 k}-2 \varphi_{i j k}+\varphi_{i j+1 k} \\
& \Delta z^{2} \delta_{z}^{2} \varphi_{i j k}=\varphi_{i j k+1}-2 \varphi_{i j k}+\varphi_{i j k-1} \\
& 2 \Delta x \delta_{x} \varphi_{i j k}=\varphi_{i+1 j k}-\varphi_{i-1 j k} \\
& 2 \Delta y \delta_{y} \varphi_{i j k}=\varphi_{i j+1 k}-\varphi_{i j-1 k} \\
& 2 \Delta z \delta_{z} \varphi_{i j k}=\varphi_{i j k+1}-\varphi_{i j k-1}
\end{aligned}
$$

The diffusion term is computed implicitly, while the advection and the nonlinear terms are calculated explicitly. The scheme is unconditionally stable for linear diffusion and second-order accurate in space. However, it is not an efficient technique to obtain the steady-state solution for fine spatial grids and/or fast transient phenomenon. An efficient fast and unconditionally stable algorithm for solving diffusion equation is presented in ref. 28. A small positive parameter $\gamma$ is introduced to accelerate the convergence of the original scheme as follows:

$$
\begin{gathered}
\left(1-\frac{1+\gamma}{2} r_{x} \delta_{x}^{2}\right)\left(\varphi_{i j k}^{n+1 / 3}-\varphi_{i j k}^{n}\right)=\left(r_{x} \delta_{x}^{2}+r_{y} \delta_{y}^{2}+r_{z} \delta_{z}^{2}\right) \varphi_{i j k}^{n} \\
+\frac{6 \gamma+2 \gamma^{3}}{8} r_{x} r_{y} r_{z} \delta_{x}^{2} \delta_{y}^{2} \delta_{z}^{2} \varphi_{i j k}^{n}+\Delta t \tilde{\rho}_{i j k}^{n} . \\
\left(\varphi_{i j k}^{n+2 / 3}-\varphi_{i j k}^{n+1 / 3}\right)=\frac{1+\gamma}{2} r_{y} \delta_{y}^{2}\left(\varphi_{i j k}^{n+2 / 3}-\varphi_{i j k}^{n}\right) \\
\times\left(\varphi_{i j k}^{n+1}-\varphi_{i j k}^{n+2 / 3}\right)=\frac{1+\gamma}{2} r_{z} \delta_{z}^{2}\left(\varphi_{i j k}^{n+1}-\varphi_{i j k}^{n}\right) .
\end{gathered}
$$

The leading truncation error term in this method is $O\left(\Delta x^{2}+\right.$ $\left.\Delta y^{2}+\Delta z^{2}+\gamma \Delta t\right)$. The parameter $\gamma$ compromises accuracy; however, it eliminates the oscillations due to large time steps. As we are interested in the steady-state solution, this approach satisfies our need to have large time steps. As suggested in ref. 28, we take $0.02<\gamma<0.05$. We implemented the above scheme for solving the PB equation. The first step in (15) only involves the diffusion operator in the $x$-direction. Therefore, the resulting set of algebraic equations is tridiagonal. Similarly, the second and third steps involve the diffusion operators in the $y$ - and $z$-directions, respectively. We use Thomas Algorithm to solve the resulting tridiagonal linear systems efficiently. ${ }^{19}$ The number of operations in this method is proportional to the number of unknowns. Therefore, the number of operations of the method proposed here is also proportional to the number of unknowns (Fig. 1b). Note that 3D elliptic solvers result in matrices with much larger bandwidths. Even efficient iterative solvers such as the preconditioned conjugate gradient solver scales with $N \log N$. In this approach, four variables $\left(\varphi^{n}, \varphi^{n+1 / 3}, \varphi^{n+2 / 3}\right.$, and $\left.\varphi^{n+1}\right)$ are needed in the calculations. However, we were able to implement this approach by using two variables accomplishing a storage requirement identical to the explicit FTCS scheme (Table 1). After solving for the $x$-direction in (15), the results for $\varphi^{n+1 / 3}$ are stored in a dummy array. The second step in (15) does not involve derivatives of 

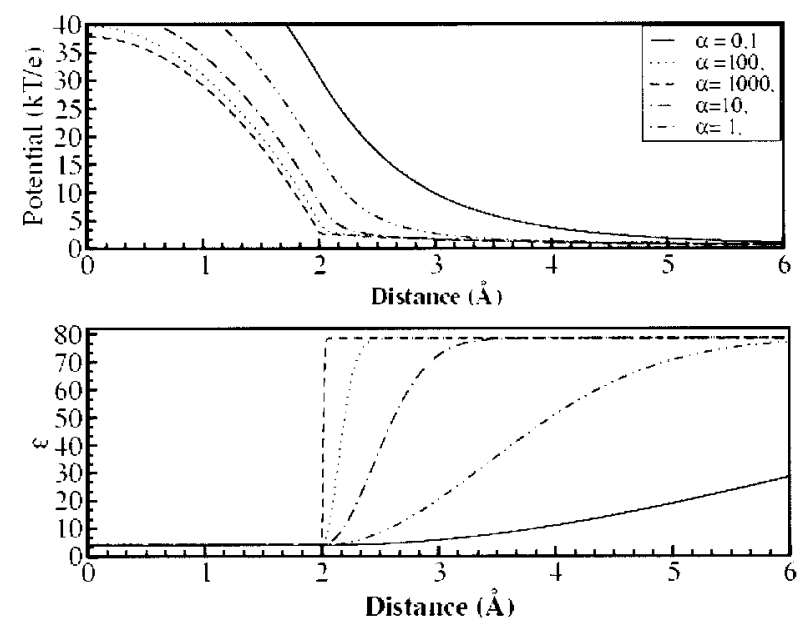

Figure 2. The effect of dielectric constant profile on the electrostatic potential distribution for a single atom immersed in a $0.1 \mathrm{M}, 1: 1$ electrolyte.

$\varphi^{n+1 / 3}$. Therefore, $\varphi^{n+2 / 3}$ is overwritten on the same dummy array. Similarly, solution for $\varphi^{n+1}$ does not involve derivatives of $\varphi^{n+2 / 3}$. Furthermore, it only involves derivative of $\varphi^{n}$ with respect to $z$. Therefore, as the solution for $\varphi^{n+1}$ is obtained, one can replace the dummy variable with $\varphi^{n}$ and the array used to store $\varphi^{n}$ with $\varphi^{n+1}$. This implementation not only eliminates two arrays, but also allows one to recover and go back to the previous time step, in case a numerical instability is encountered. In summary, 16 $N$ (using double precision) storage is needed for $\left(\varphi^{n}, \varphi^{n+1 / 3}\right.$, $\varphi^{n+2 / 3}$, and $\left.\varphi^{n+1}\right)$.

\section{Spatial Variations of the Dielectric Constant}

The dielectric constant in most biomolecular systems is generally assumed to have two components. At points in the aqueous medium, a local electrical field tends to align the water molecules. This has the effect of spreading out the charge density associated with a fixed ion, thereby decreasing the electrical field. Within the atoms of the embedded structure the electron cloud is distorted, again countering a nearby field. These effects are accounted for in most PB theories by taking $\varepsilon(\vec{r})$ to have its bulk value in the aqueous medium and to be distinct within the atoms of the immersed structure. To be sure, the dielectric constant must change continuously from its bulk aqueous medium value to the intraatom value. This transition should be smooth in that the electron charge density changes continuously and that a mesoscopic model like the PB equation should reflect some influence of the fluctuating locations of the atoms of the embedded structure.

In this study we use the following expression to approximate the smooth transitional variation in the dielectric constant:

$$
\varepsilon(\vec{r})=\min _{i=1, \ldots, N_{\text {atoms }}}\left\{\varepsilon_{m}+\left(\varepsilon_{\text {atom }}-\varepsilon_{m}\right) \exp \left(-\alpha\left(\left|\vec{r}-\vec{r}_{i}\right|-R_{i}\right)^{n} / R_{i}^{n}\right)\right\}
$$

where $\varepsilon_{m}$ and $\varepsilon_{\text {atom }}$ are the dielectric constants of the medium and atom, respectively, $\alpha$ and $n$ are phenomenological constants, $R_{i}$ is the radius of atom $i$, and $\vec{r}_{i}$ is the position of the center of atom $i$.

The effect of the dielectric constant distribution on the potential for a spherically symmetric system is shown in Figure 2. The grid spacing in this one-dimensional (spherically symmetric) simulation is $0.001 \AA$ to capture the discontinuity of the dielectric constant at the surface of the atom that arises for large values of $\alpha$ and $n$, while smoother variations are also shown. Capturing the discontinuity requires either extremely small grid spacing or an adaptive grid approach for accurate solutions. ${ }^{29}$ However, as discussed above, this is an artifact of a step function approximation for the dielectric constant. Thus, in this study, $R_{i}$ is taken to be atom-oxygen van der Waals as obtained from CHARMM22 force field, $\alpha$ and $n$ are taken as 1 and 2, respectively, reducing (17) to a Gaussian smoothing function. We use a grid spacing of $0.3-0.6$ $\AA$, which allows for the solution of fairly large macromolecules (over $200 \AA$ in size) on a single processor computer with 3 GB of RAM.

\section{Numerical Results}

In this section, we demonstrate the accuracy of our simulator and its application to large molecular systems. First consider a uniformly charged spherical particle of $2 \AA$ radius and total charge of $+1 \mathrm{e}$ immersed in 1:1 electrolyte. This model has been used to study globular proteins in solution. ${ }^{8}$ The $\mathrm{PB}$ equation in spherical coordinates can be written

$$
\begin{aligned}
\frac{1}{r^{2}} \frac{d}{d r}\left(\varepsilon(r) r^{2} \frac{d \varphi}{d r}\right)+4 \pi F \sum_{i=1}^{N_{\text {ions }}} z_{i} c_{i}^{\infty} \exp \left(-z_{i} F \varphi / R T\right) & \\
+ & \frac{4 \pi q}{V} \vartheta(r)=0
\end{aligned}
$$

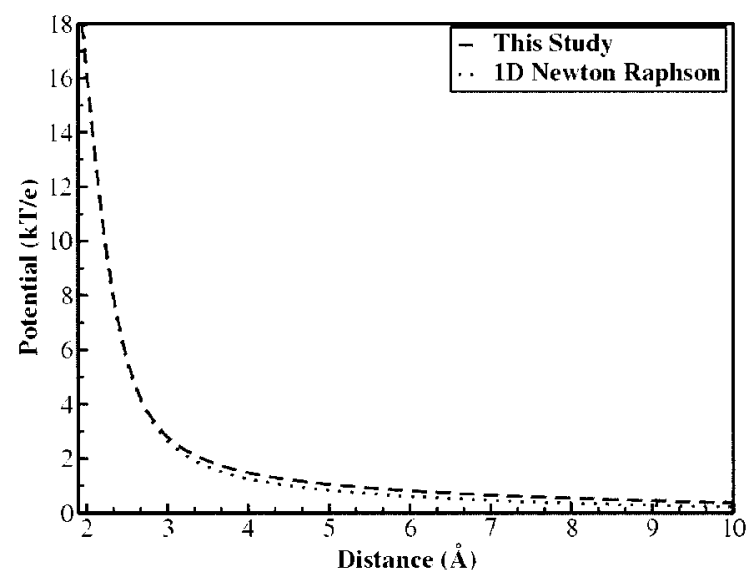

Figure 3. Electrostatic potential for a spherical particle of uniform charge density and total charge of $+1 \mathrm{e}$ immersed in a 1:1 electrolyte of ionic strength $0.1 \mathrm{M}$. A highly accurate $(0.001 \AA$ spacing) NewtonRaphson 1D solver agrees well with the solution obtained using our 3D solver (0.15 ̊ grid spacing). 

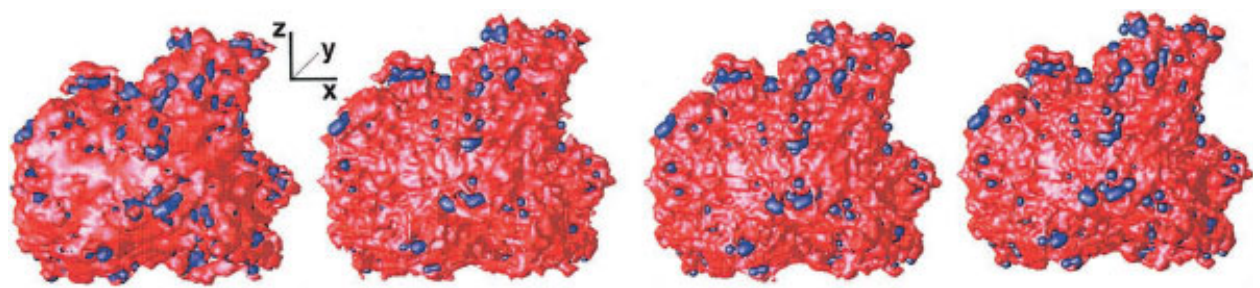

Figure 4. Electrostatic potential isosurfaces of $+5 \mathrm{kT} / \mathrm{e}$ (red) and $-10 \mathrm{kT} / \mathrm{e}$ (blue) of polio virus protomer (11,893 atoms) immersed in a $0.1 \mathrm{M}, 1: 1$ electrolyte for $151^{3}, 201^{3}, 251^{3}$, and $301^{3}$ finite difference grids. As the resolution is enhanced, a smoother isosurface is obtained. There appears to be little difference between the two fine grid simulations.

where

$$
\vartheta(r)= \begin{cases}1, & r<r_{0} \\ 0, & r>r_{0}\end{cases}
$$

and $r_{0}$ is the radius of the particle with volume $V$. The underlying physical picture of the charge distribution within an atom is implied by the wave function of the electrons associated with atoms. ${ }^{30}$ With this, the charge density as a function of space is calculated as the sum of the localized nuclear charge and the electron density around it.

To verify our PB simulator, we have solved (18) using a finite difference scheme with the Newton-Raphson method to solve the nonlinear one-dimensional PB equation. The solution of our 3D solver agrees well with the 1D Newton-Raphson solver (Fig. 3). The 3D grid spacing was $0.15 \AA$ and the grid spacing of the $1 \mathrm{D}$ solution was $0.001 \AA$.

The objective of our approach is to obtain high-resolution PB simulations to capture the electrostatic potential around macromolecular and other mesoscopic systems. The capsid of the polio virus consists of 12 pentamers, each of which are made of five protomers. The protomer consists of three proteins, one of which is cleaved to yield a four-protein, mature protomer. To demonstrate the applicability of our method to large systems, we choose the immature capsid components. The protomer consists of 11,893 atoms. Fractional charges were assigned according to the CHARMM22 force field. The structure of the protomer and pentamer were that at the energy minimum as determined by the VirusX macromolecular mechanics code. ${ }^{31}$

To test the consistency of the numerical technique, we solve the electrostatic potential for the protomer at various finite difference resolutions. The protomer is immersed in a $0.1 \mathrm{M}, 1: 1$ electrolyte. Figure 4 shows the electrostatic potential surfaces for $+5 \mathrm{kT} / \mathrm{e}$ (red) and $-10 \mathrm{kT} / \mathrm{e}$ (blue) for $\left(151^{3}, 0.9 \AA\right.$ grid spacing), $201^{3}$,
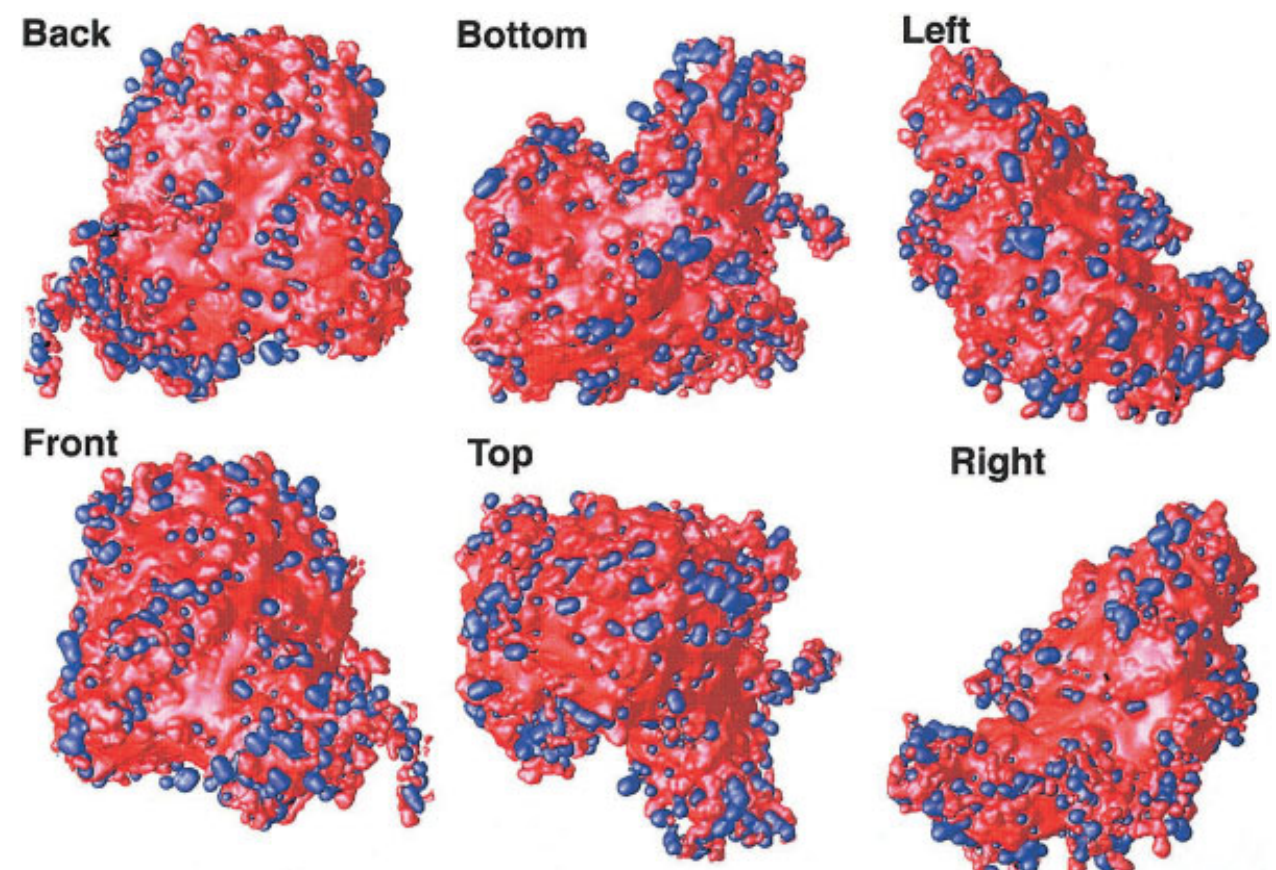

Right

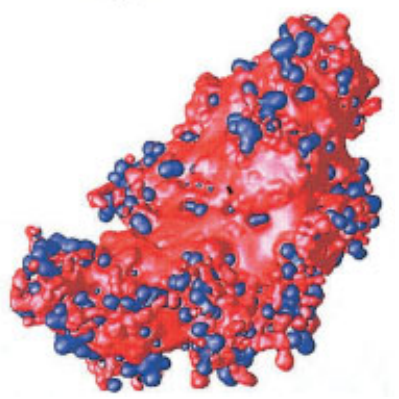

Figure 5. Six views of electrostatic potential isosurfaces of $+5 \mathrm{kT} / \mathrm{e}$ (red) and $-10 \mathrm{kT} / \mathrm{e}$ (blue) of polio virus monomer (11,893 atoms) immersed in a $0.1 \mathrm{M}, 1: 1$ electrolyte. 


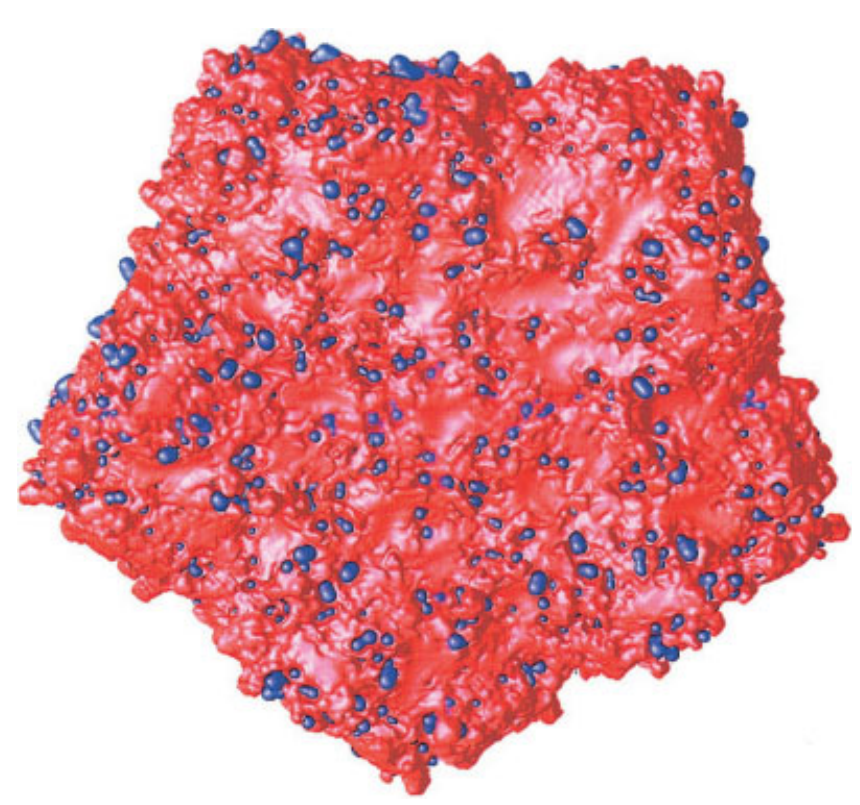

Figure 6. Electrostatic potential isosurfaces of $+5 \mathrm{kT} / \mathrm{e}$ (red) and -10 $\mathrm{kT} / \mathrm{e}$ (blue) of polio virus pentamer (59,465 atoms) immersed in a 0.1 M, 1:1 electrolyte. [Color figure can be viewed in the online issue, which is available at www.interscience.wiley.com.]

$251^{3}$, and $301^{3}$ regular finite difference grids. As expected, there is negligible difference between the two fine resolution simulations, demonstrating the numerical consistency of the method. The results were obtained to an accuracy of $0.01 \mathrm{kT} / \mathrm{e}$. The $301^{3}$ grid simulation took approximately $15 \mathrm{~min}$ on a $2.6-\mathrm{GHz}$ Pentium IV computer. Figure 5 shows the same isosurfaces from different angles of view for the highest resolution grid.

The electrostatic potential for the pentamer required a larger grid $(632 \times 596 \times 417,0.33 \AA$ grid spacing). Thus, the potential was determined at 155 million grid nodes. Obtaining the solution to an accuracy of $0.01 \mathrm{kT} / \mathrm{e}$ required approximately $10 \mathrm{~h}$ of CPU time on a single processor of the IBM SP power4 computer located at Indiana University (Fig. 6). The fivefold symmetry in Figure 6 shows that the numerical resolution was sufficient in this particular case. The difference in the field profiles between the free protomer and that as a part of the pentamer are closely relation to changes in conformation near the protomer-protomer interfaces. This illustrates the importance of carrying out the PB computations for large systems even when only local effects are of interest (e.g., for drug discovery).

\section{Conclusion}

An efficient numerical method for the calculation of the electrostatic potential based on the nonlinear PB equation has been developed. Our methodology allows us to simulate larger molecular structures than previously possible. This efficiency will allow simulating the electrostatics of complex systems such as enzymes, ribosomes, and viruses. Furthermore, it will make it possible to cure the difficulties in the $1 / r$ potential seen in molecular dynamics/mechanics simulations based on CHARMM22 or other force fields. This will, however, require some recalibration of these force fields. A difficulty in existing PB solvers is that the classic $\mathrm{PB}$ equation is based on the assumption that the chemical potential of the mobile ions can be approximated by the dilute solution theory. This leads to an unphysical exponential nonlinearity in the PB equation with consequent overestimate of mobile ion concentrations near the fixed charges. As our methodology is computationally efficient, we shall generalize the approach by solving equilibrium equations for each mobile ion concentration that reflect the nonideality, simultaneously with a Poisson equation for the electrostatic potential. Our PB simulator is expected to be available at http://bl-chem-pb.chem.indiana.edu by early 2004 .

\section{References}

1. Sharp, K.; Honig, B. Annu Rev Biophys Chem 1990, 19, 301.

2. Schultz, C. N.; Warshel, A. Proteins Struct Funct Genet 2001, 44, 400.

3. Gilson, M. K.; McCammon, J. A.; Madura, J. D. J Comput Chem 1995, 16, 1081.

4. McQuarrie, D. A. Statistical Mechanics; Harper Collins: New York, 1976.

5. Levine, S.; Bell, G. M. J Phys Chem 1960, 64, 1188.

6. Borkukhov, I.; Andelman, D.; Orland, H. Phys Rev Lett 1997, 79, 435.

7. Guarnieri, F.; Schmidt, A. B.; Mehler, E. L. Intl J Quantum Chem 1998, 69, 57.

8. Tanford, C. Physical Chemistry of Macromolecules; John Wiley and Sons: New York, 1961.

9. Shklovskii, B. I. Phys Rev E 1999, 60, 5802.

10. Ortoleva, P. J. Mesoscopic Chem Phys 2003.

11. Fuoss, R. M.; Katchalsky, A.; Lifson, S. Proc Natl Acad Sci USA 1951, 37, 579.

12. Coalson, R. D.; Beck, T. L. In Encyclopedia of Computational Chemistry; Schleyer, P. v. R., Ed.; John Wiley: New York, 1998, p. 2086.

13. David, L.; Field, M. J. J Comput Chem 1997, 18, 343.

14. Vorobjev, Y.; Scheraga, H. J Comput Chem 1997, 18, 569.

15. Bordner, A. J.; Huber, G. A. J Comput Chem 2003, 24, 353.

16. Nicholls, A.; Honig, B. J Comput Chem 1991, 12, 435.

17. Bruccoleri, R. E.; Novotony, J.; Davis, M. E.; Sharp, K. A. J Comput Chem 1997, 2, 268.

18. Grant, J. A.; Pickup, B.; Nicholls, A. J Comput Chem 2001, 22, 608.

19. Fletcher, C. A. J. Computational Techniques for Fluid Dynamics; Springer-Verlag: Berlin, 1988.

20. Holst, M.; Saied, F. J Comput Chem 1993, 14, 105.

21. Tomac, S.; Gräslund, A. J Comput Chem 1998, 19, 893.

22. Shestakov, A. I.; Milovich, J. L.; Noy, A. J Collid Interface Sci 2002, 247, 62 .

23. Jackson, J. D. Classical Electrodynamics; Wiley: New York, 1975.

24. Landau, L. D.; Lifshitz, E. M. Electrodynamics of Continuous Media; Pergamon: New York, 1960.

25. MacKerell, A. D., Jr., Bashford, D.; Bellot, M.; Dunbrack, R. L., Jr.; Evanseck, J. D.; Field, M. J.; Fischer, S.; Gao, J.; Guo, H.; Ha, S.; Joseph-McCarthy, D.; Kuchnir, L.; Kuczera, K.; Lau F. T. K.; Mattos, C.; Michnick, S.; Ngo, T.; Nguyen, D. T.; Prodhom, B.; Reiher, W. E., III; Roux, B.; Schlenkrich, M.; Smith, J. C.; Stote, R.; Straub, J.; Watanabe, M.; Wiórkiewicz-Kuczera, J.; Yin, D.; Karplus, M. J Phys Chem B 1998, 102, 3586.

26. Hill, T. L. An Introduction to Statistical Thermodynamics; Dover Publications: New York, 1986.

27. Douglas, J. J.; Gunn, J. E. Numer Math 1964, 6, 428.

28. Dai, W.; Nassar, R. Intl J Numerical Methods Heat Fluid Flow 1997, 7, 659.

29. Baker, N.; Holst, M.; Wang, F. J Comput Chem 2000, 21, 1319.

30. Politzer, P.; Murray, J. S.; Lane, P. J Comput Chem 2003, 24, 505.

31. Jarymowycz, L.; Hubbard, K.; Tuncay, K.; Ortoleva, P., in preparation. 